\title{
Online State Of Health Estimation of Lithium-Ion batteries based on improved Ampere-Count Method
}

\author{
Edoardo Locorotondo, Luca Pugi, Lorenzo Berzi, Marco Pierini \\ Department of Industrial Engineering \\ University of Florence \\ Florence, Italy \\ edoardo.locorotondo@unifi.it, luca.pugi@unifi.it, \\ lorenzo.berzi@unifi.it, marco.pierini@unifi.it
}

\author{
Alessandro Pretto \\ Gruppo Pretto \\ GP \\ Ponsacco, Italy \\ alepretto@gruppopretto.it
}

\begin{abstract}
State Of Health (SOH) is one of the most important parameters in order to evaluate battery performances over time and it has profound impact on performances, cost and reliability of the target vehicle. SOH definition requires an accurate estimation of the actual battery energy storage capacity at low frequencies. This paper presents an improved AmpereCount Method based on Incremental Capacity Analysis in a particular voltage range delivered by battery for SOH estimation. Proposed method is implemented using Matlab Software, and its effectiveness has been verified based on simulated data from thermo-electrical model of $\mathrm{LiFePO} 4$ cell with aging phenomena realized on Siemens Amesim software. Model was calibrated with data provided by manufacturer and based on real data acquired from LiFePO4 battery packs installed on a Electric Vehicle (EV).
\end{abstract}

Keywords-battery; lithium-ion; online SOH estimation; Ampere-Count; Incremental Capacity Analysis; electric vehicles;

\section{INTRODUCTION}

Analysis, modeling and management of energy storages in the batteries, mainly Lithium-Ion types, are topics discussed in major European research programs (Horizon 2020) and in automotive industry. Battery performances vary significantly with age and so it is important to represent these features and to synthesize algorithms to predict its End Of Life (EOL). Aging should be described by a parameter defined State Of Health ( $\mathrm{SOH})$. This is usually represented as a vector that contains battery parameters which degrade over time, such as:

- Residual capacity to store energy.

- Internal resistance.

- Maximum discharging peak power.

- Open Circuit Voltage (OCV) - State Of Charge (SOC) relationship.

In this work, actual battery energy storage capacity is represented as indicator of $\mathrm{SOH}$. Consequently, battery $\mathrm{SOH}$ is defined as the following equation:

$$
S O H=\frac{Q_{\text {aged }}[A h]}{Q_{\text {new }}[A h]} \times 100 \%
$$

where Ah indicates the charge transferred by a steady current flowing for one hour; $Q_{\text {aged }}$ indicates the maximum available capacity in current state and $Q_{n e w}$ indicates the nominal battery capacity. The residual capacity can be computed by a normal Ampere-Count method, which is based on recursive calculation of the integral of the current delivered by the battery over time, through a defined number of complete battery standard charge and discharge (at constant current discharge rates) phases, as suggested by the International standards ISO 12405-1,2 [1], [2] and IEC 62660-1 [3]. However this solution should be used only in laboratory tests. Indeed, in real operating conditions, charge and discharge phase is often partial or not complete. Hence, the online Ampere-Count method must focus in a particular range of SOC when the Electric Vehicle (EV) is usually used. In Fig. 1 is represented Ah-V curve (SOC is replaced by accumulated capacity in $A h$ ) of LiFePO4 cell at different SOH. From Fig. 1 it's clearly noticeable that Ah-V curve exhibits some particular features which are quite not sensitive respect to battery SOH. In this way, it's possible to identify a known SOC level, recognizing a specific feature (e.g. feature highlighted in Fig. 1 is the knee of the Ah- $\mathrm{V}$ curve localized above about 3.3V). This work presents an improved Ampere-Count Method based on Incremental Capacity Analysis (ICA) [4], [5] of this particular feature. The reasons why SOH estimation is carried out on this feature are mainly three:

1) This feature is always present during cell's life: as specified previously.

2) Possibility to apply algorithm during each recharge cycle of Electric Vehicle $(\mathbf{E V})$ : estimating $\mathrm{SOH}$ in a SOC range of $[65,95] \%$, which is often achievable along a common EV cycle.

3) Relationship between SOH-Feature's slope: aged cell, as shown in Fig. 1, assumes a more flat curve than new cell, in particular in this specific feature (good for ICA).

Performances of the proposed method have been verified on simulated data from a thermo-electrical model of LiFePO4 cell with aging behaviors realized on Siemens Amesim software, shown in Fig. 2.

\section{CELl THERMO-ELECTRICAL MODEL}

The Lithium-Ion cell model (Fig. 2) simulated in this work is a prismatic $\mathrm{LiFePO} 4$ cell manufactured by China Aviation Lithium Battery (CALB). 


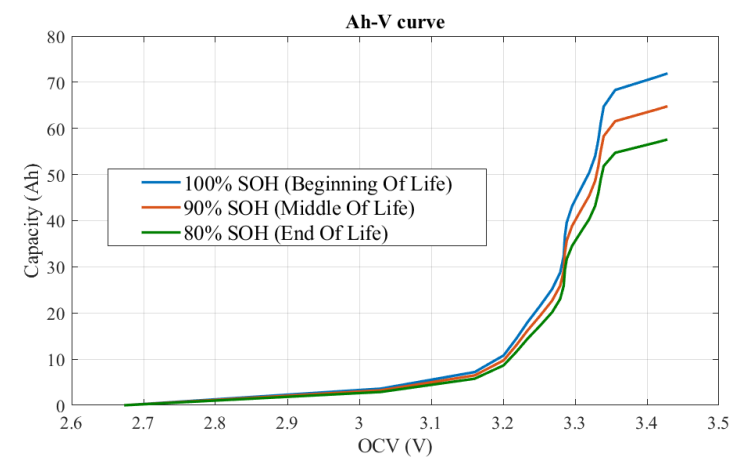

Fig. 1. Ah-V curves of LiFePO4 cell model simulated on Siemens Amesim at different SOHs and the particular feature's curves analyzed in this work.

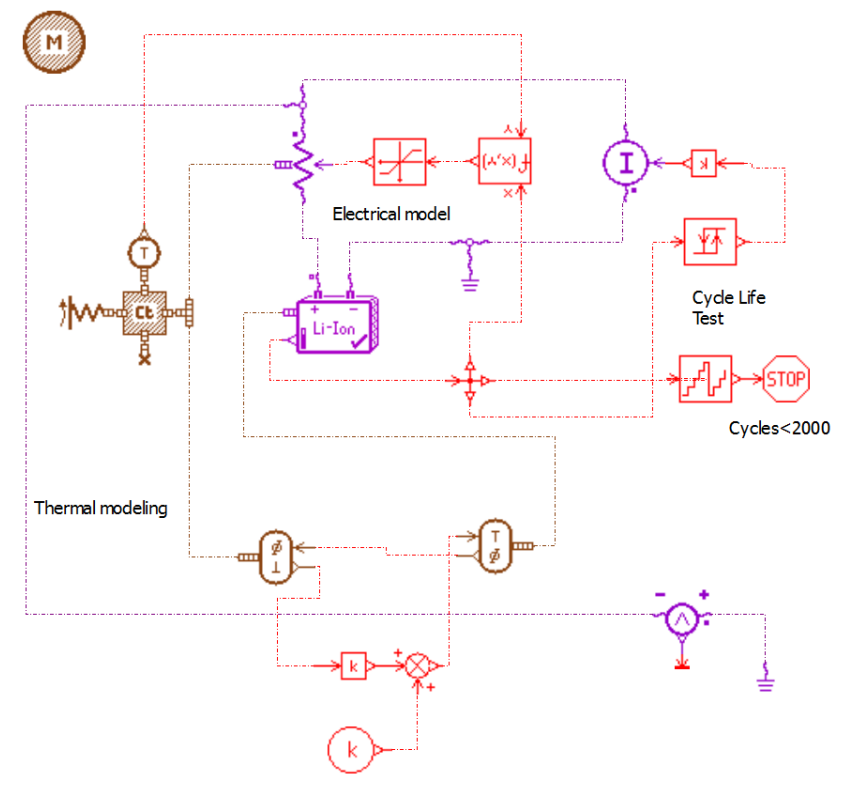

Fig. 2. Cell thermo-electric model in Siemens Amesim: ready to test its cycle life.

This cell has $72 \mathrm{Ah}$ of nominal capacity and $3.2 \mathrm{~V}$ of nominal voltage $(\mathrm{dc})$. To replicate its thermal and electrical behavior, the LiFePO4 cell quasi-static model is chosen from electric storage's library on Siemens Amesim. Parameters of this model, which takes into account aging phenomena, have been calibrated and validated on experimental tests carried out by the research institute IFP Energies nouvelles [6], [7], [8]. Single cell model should have nominal capacity in the range $[2.3,8]$ Ah. In this work, battery pack is chosen: it's composed by 18 cells in parallel with 4 Ah of rated capacity and $3.2 \mathrm{~V}$ of nominal voltage. Then, a variable resistance $\left(R_{i n t}\right)$ is inserted in series to the cell model in order to simulate voltage drops and heat dissipations produced by the internal resistance of the real cell. In particular, value of internal resistance depends from actual SOC of cell and the surrounding temperature. In [9] is realized an appropriately surface function $R_{\text {int }}=$ $\Upsilon(S O C, T)$ which returns the value of the internal resistance

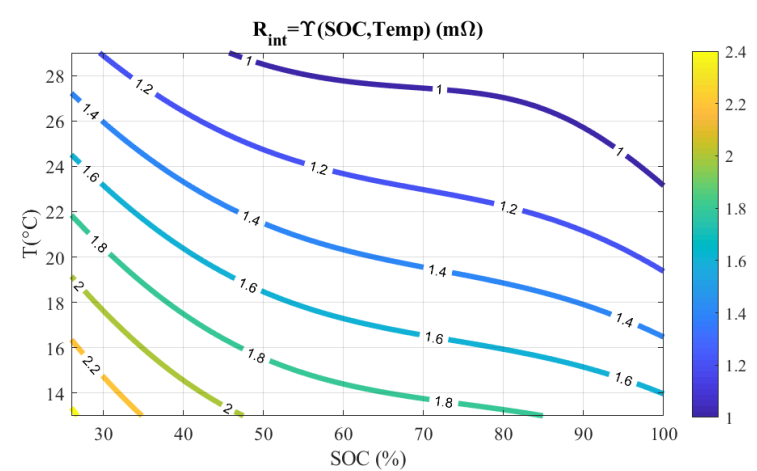

Fig. 3. Analytical surface function $\Upsilon$ for single LiFePO4 cell (battery pack monitored is composed by 30 cell in series): contour plot.

$R_{i n t}$ in function of the SOC and the surrounding temperature. Coefficients of this surface function are sized using Curve Fitting Toolbox of Matlab, and are identified based on real data acquired from LiFePO4 battery packs (composed by 30 CALB cells in series) installed on a EV and online estimation algorithms shown in [9]. In Fig. 3 is shown contour plot of internal resistance surface function. LiFePO4 cell quasistatic model and variable resistance complete electrical model of battery. Then, thermal cell model is realized as follows: referring to [10], [11], equivalent electrical circuit model shown in Fig. 4 should describe thermal dynamics from inside to outside of the cell. Indeed, thermal and electrical processes represent exchanges of energy flows between two physical entities; so there is a strong correlation in the description of the two physical dynamics. After this consideration, the difference of temperature should be associated with a difference of electrical potential of two sources on a physical conductor; moreover the heat flow should be associated with the current flow exchanged between these. Cell thermal model is shown in Fig. 4 illustrates the dynamics of the exchange of heat flows from inside the cell to the outside. $T_{i n}$ indicates the cell internal temperature; the amount of heat dissipated inside for Joule effect corresponds to the ideal current generator $Q_{t h, i n}$. Finally, transient energy exchanges between internal and surface of the cell are represented by the same electric dynamics of an RC unit.

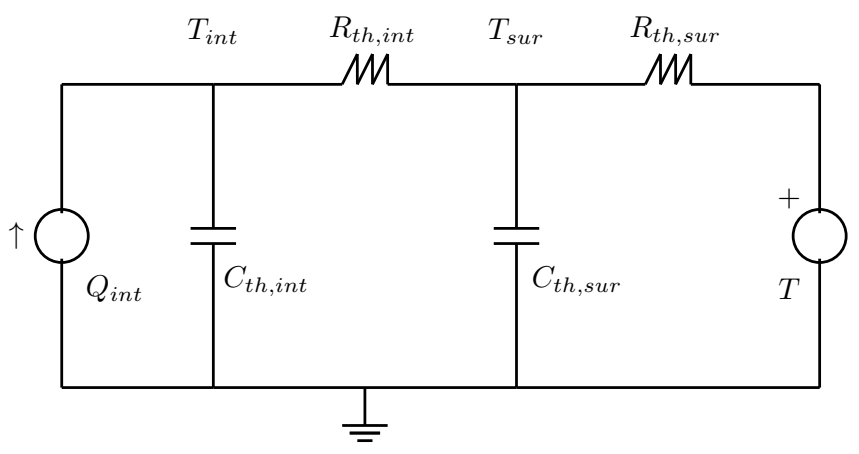

Fig. 4. Cell thermal model. 
It's composed by $C_{t h, i n t}$, which is cell internal thermal capacity, and $R_{t h, i n t}$, which is inversely proportional to heat conduction coefficient between internal and cell surface. $T_{\text {sur }}$ indicates cell surface temperature. Exchanges of heat flows between the surface $\left(T_{\text {sur }}\right)$ of the cell and the surrounding environment $(T)$ are represented by $R_{t h, s u r}, C_{t h, s u r}$ parameters. Thermal parameters are sized during phase recharging, analyzing real internal temperature data acquired on battery packs [9]. In particular thermal capacity and resistance are sized using thermodynamics equations:

$$
C_{\text {thermal }}=m c, \quad R_{\text {thermal }}=\frac{S}{\lambda}
$$

$m$ is the mass of the cell; $c$ is the specific heat; $S$ is the surface of the cell invested by the fluid; $\lambda$ is the thermal conductivity coefficient. Finally, as its seen in Fig. 2, another heat source is inserted in parallel to thermal model to add all heat dissipation transient effects shown from equivalent thermal circuit represented in Fig. 4, and in [10], [11]. In Table I are listed values which are signed to thermo-electrical cell model. Cycle life test is applied to verify cell capacity's degradation and it consists of two phases:

1) Cell standard charge: charging is performed up to 3.40 $\mathrm{V}$ at a constant current of $21.6 \mathrm{~A}$; the value is reduced continously in order to mantain the voltage to $3.40 \mathrm{~V}$ until the current was not more than $3.6 \pm 0.5 \mathrm{~A}$, at room temperature $\left(25^{\circ} \mathrm{C}\right)$.

2) Cell standard discharge: a discharge current of $21.6 \mathrm{~A}$ is imposed considering a cut-off voltage $(2.6 \mathrm{~V})$ and a room temperature $\left(25^{\circ} \mathrm{C}\right)$.

These two steps (cycle) are repeated until the discharge capacity reached the $80 \%$ of rated capacity; the number of cycles completed defines the battery cycle life. Cell thermoelectrical model is chosen as in Table I and cell degradation curve of capacity is shown and compared with real CALB cell degradation in Fig. 5. It's clearly noticeable a quasiperfect tracking between two curves. So this cell thermoelectric model implemented in Amesim validates successfully CALB degradation of capacity in its cycle life.

TABLE I

CELL THERMO-ELECTRIC MODEL'S PARAMETER VALUES INSERT FOR THE SIMULATION OF REAL PRISMATIC CALB CELL

\begin{tabular}{|l|l|}
\hline \multicolumn{1}{|c|}{ Parameter } & \multicolumn{1}{c|}{ Value } \\
\hline Weigth cell & $1.9 \mathrm{~kg}$ \\
Volume cell & $869 \mathrm{~cm}^{3}$ \\
Nominal voltage & $3.2 \mathrm{~V}$ \\
Nominal capacity & $72 \mathrm{Ah}$ \\
Thermal resistance & $0.06 \mathrm{~K} / \mathrm{W}$ \\
Thermal capacity & $1050 \mathrm{~J} / \mathrm{K}$ \\
Room temperature & $25^{\circ} \mathrm{C}$ \\
Velocity fluid & $1.5 \mathrm{~m} / \mathrm{s}(\mathrm{calm})$ \\
Sample time & $1 \mathrm{~s}$ \\
\hline
\end{tabular}

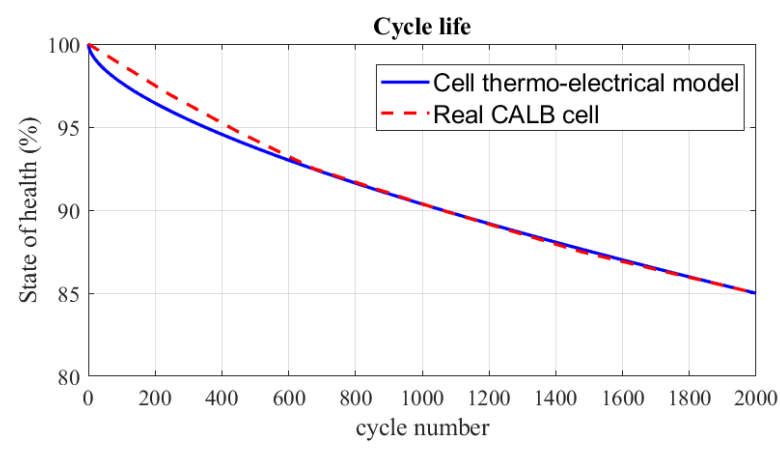

Fig. 5. Results during cycle life test: comparison between cell model and real cell.

\section{IMPROVED AMPERE-COUNT METHOD}

\section{A. Presentation}

One of the most simple way to estimate online $\mathrm{SOH}(1)$, is to use $\mathrm{Ah}-\mathrm{V}$ curve when EV is charging. Current and voltage measurements are necessary to reproduce this curve. In this work, the Ah-V curve is composed by capacity accumulated data and by Open Circuit Voltage (OCV), which is not subject to battery loads, and its relationship with SOC doesn't depend on the temperature. Indeed battery OCV changes very slowly with temperature, e.g., in [10] it was shown that OCV value changed less than $10 \mathrm{mV}$ as temperature passed from $-10^{\circ} \mathrm{C}$ to $50^{\circ} \mathrm{C}$. With current and voltage measurements, Ampere-hour throughput to battery is the integrated current over time and represents the energy stored by battery during phase charging. However, OCV is not directly measured online, but it should be accurately estimated with several filtering techniques, for example Kalman Filter [12] or other model-based observer filters. An example of cell Ah-V curve is represented in Fig. 1 at different SOHs. In particular it's noticeable that aged cell Ah-V profile assumes a more flat curve than new cell Ah$\mathrm{V}$ profile in highlighted feature. This fact implies that, by the same Ah accumulated, an aged cell must reach higher voltages compared to a new cell. The causes of this phenomena are due to electro-chemical behaviors of anode and electrolyte of the cell [4] during cell's life: the not perfect interaction between the active materials and the electrode-electrolyte interface for aged cell, consequently a possible degradation of active materials, in conclusion a reduction in the capacities of the electrodes [4]. The composition and process of film formation passive Solid Electrolyte Interphase (SEI) in the anode, during cell's life, is another process that produces a loss of capacity. In this work Incremental Capacity Analysis (ICA) is used to analyze actual cell $\mathrm{Ah}-\mathrm{V}$ profile. ICA is a mathematical method which analyzes the evolution of the Ah-V curve over time by monitoring its slope in a particular characteristic area of curve, defined feature. ICA function is defined by numeric derivative operation between two consecutive points of the AhV curve:

$$
I C A=\frac{d Q}{d V}, \quad[A h / V]
$$




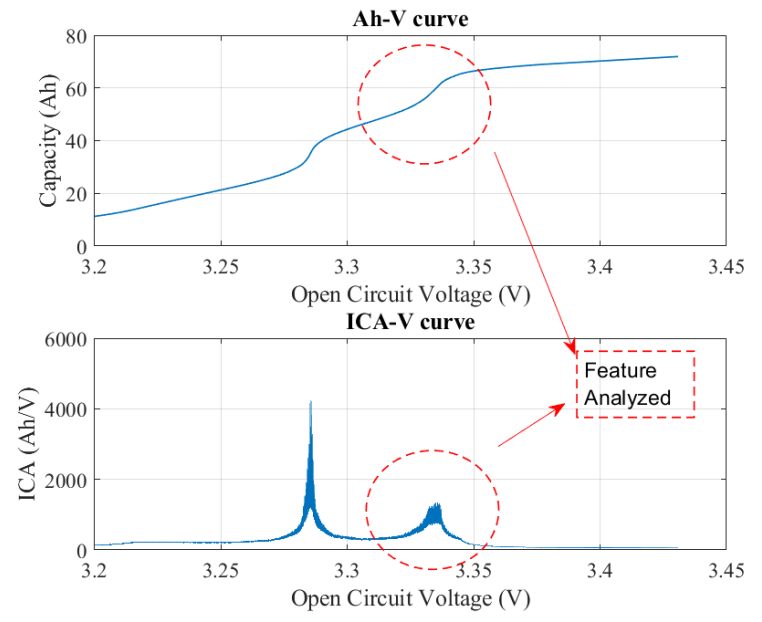

Fig. 6. ICA curve and its construction before numercal derivation.

$Q$ indicates the actual capacity accumulated by cell. CALB cell model ICA curve is represented in Fig. 6. Specific feature analyzed for CALB cell thermo-electrical model realized previously (Fig. 1 and Fig. 6) is localized in a particular voltage range $[3.31,3.36] \mathrm{V}$, which corresponds from $66 \%$ to $94 \%$ of CALB cell SOC.

\section{B. Online SOH estimation algorithm}

Once cell ICA curve is reproduced in the voltage range of interest, improved Ampere-count method is used to analyze the whole feature, i.e. integral of ICA curve is calculated. Indeed, measuring area instead of only peak maximum amplitude provides more accurate results because the integration process acts as an average of whole feature. The area under the curve between two voltage limits is equal to compute accumulated cell capacity $(\mathrm{Q})$ between these limits:

$\int_{V_{\text {inf }}}^{V_{\text {sup }}} I C A(V) d V=\int_{V_{\text {inf }}}^{V_{\text {sup }}} \frac{d Q(V)}{d V}=Q\left(V_{\text {sup }}\right)-Q\left(V_{\text {inf }}\right)$

A reduction of the area consists of a reduction in the accumulated capacity among the two voltage limits, so in proportion, a $\mathrm{SOH}$ degradation. In conclusion, computing the amount of accumulated capacity energy between $\left[V_{\text {inf }}, V_{\text {sup }}\right]$ and comparing it to new cell, the updated istantaneous $\mathrm{SOH}$ is obtained. To be able to provide a robust implementation of this algorithm, it is necessary filtering ICA signal to clean it from eventual noises. Indeed, as shown in Fig. 8, real ICA curve is perturbed by noisy signal due to process disturbances, reproducing ICA curve, and not perfect accuracy of the measuring instruments. In this work, a low-pass $2^{\text {nd }}$ order Butterworth filter is used to clean ICA curve. Magnitude of frequency response of a generic N-order's Butterworth Filter with cut frequency $\omega_{c}$ is represented by the following equation:

$$
|H(j \omega)|^{2}=\frac{1}{1+\left(\frac{\omega}{\omega_{c}}\right)^{2 N}}
$$

TABLE II

PARAMETER VALUES FOR ALGORITHM

\begin{tabular}{|l|l|}
\hline \multicolumn{1}{|c|}{ Parameter } & \multicolumn{1}{|c|}{ Value } \\
\hline Sample time & $1 \mathrm{~s}$ \\
Input costant current recharge & $21.6 \mathrm{~A}$ \\
Input current disturb & $\pm 0.5 \mathrm{~A}$ \\
Accuracy voltage measurement & $\pm 1 \mathrm{mV}$ \\
Voltage range analyzed & {$[3.31,3.36] \mathrm{V}$} \\
Resolution (s. frequency) feature & $0.015 \mathrm{mV}(67.04 \mathrm{kHz})$ \\
Cut frequency Butterworth & $700 \mathrm{~Hz}$ \\
Initial condition of Butterworth $1^{\text {st }}$ order & $300 \mathrm{Ah} / \mathrm{V}$ \\
Initial condition of Butterworth $2^{\text {nd }}$ order & $-275 \mathrm{Ah} / \mathrm{V}^{2}$ \\
\hline
\end{tabular}

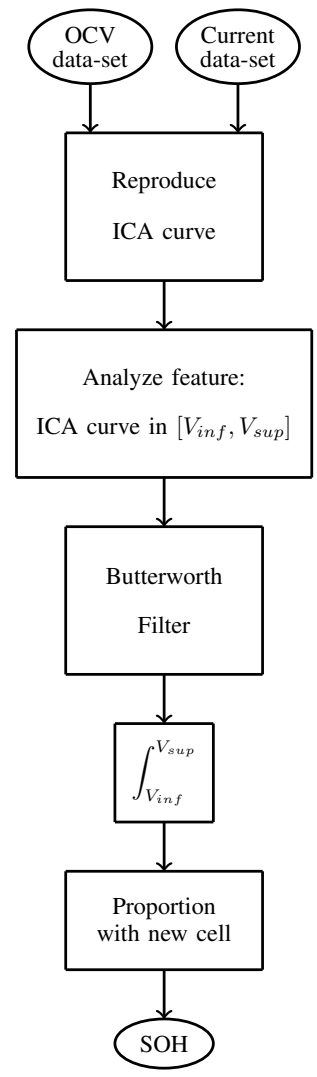

Fig. 7. SOH estimation represented on a flow-chart.

This type of filter is chosen because has the most flat magnitude response in the pass-band respect to other filters and using order's filter $N=2$, the attenuation beyond the cutoff frequency is a moderately steep $-40 \mathrm{~dB} /$ decade and is acceptable for this work. Finally only cut-off frequency and initial conditions of Butterworth filter $(\mathrm{dAh} / \mathrm{dV}$ and its derivative) should be tuned. Results of ICA filtered are shown in Fig. 8. Now, improved Ampere-Count algorithm is complete. This algorithm is realized using Matlab software, because Butterworth filter is easily implementable thanks to Signal Processing Toolbox in Matlab. Parameter values for algorithm are shown in Table II and a summary of improved AmpereCount method for $\mathrm{SOH}$ estimation is represented on a flowchart in Fig. 7. 


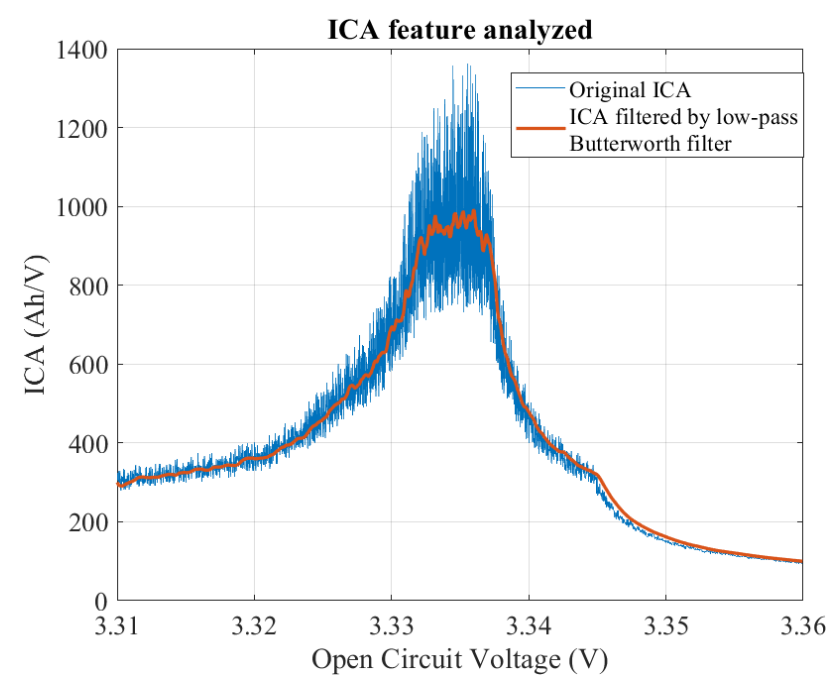

Fig. 8. ICA curve reproduced by Butterworth filter.

\section{RESULTS}

SOH estimation's effectiveness using improved AmpereCount method is evaluated during many recharging test simulations on cell thermo-electric model implemented in Siemens Amesim and shown in Fig. 2. Firstly accumulated capacity for a new cell $(100 \% \mathrm{SOH})$ in voltage range $\left[V_{\text {inf }}, V_{\text {sup }}\right]$ (in this work is chosen $[3.31,3.36] \mathrm{V}$ ) is defined using Ampere count method. After this capacity calibration test, 100 recharge tests are performed to assess a more robust evaluation on the improved Ampere Count algorithm's effectiveness. Input charge current is supplied to cell based on standard charge described in the previous section; an input current disturb is added to it: this additive disturb is modeled as a white noise with zero mean and its standard deviation value is equal to input current disturb value shown in Table II. Moreover, a white noise voltage disturb is added during voltage measurement, and its standard deviation value is shown in Table II. Algorithm is tested to different cell SOHs, exactly to $100 \%$, $99,5 \%, 99 \%, 95 \%, 90 \%, 85 \%, 80 \%$. For each defined cell $\mathrm{SOH}, 100$ recharge tests are performed: during these tests, current and voltage data changes due to the additive noise. Filtered ICA curve at different $\mathrm{SOH}$ is shown in Fig. 9. As expected, ICA curve of aged cell is lower than new cell in all voltage range. This result proves a lower capacity accumulated by aged cell at the same voltage difference, thus a real capacity loss. All Results of cell SOH estimation are shown in Table III, after 100 recharge trials. It is noted that the estimated $\mathrm{SOH}$ corresponds to its expected with a little standard deviation, which defines the accuracy of the SOH estimation method. Therefore we observe in Table III that results slightly deviate from the mean value, which is approximately equal to the real $\mathrm{SOH}$ value, so effectiveness of the improved Ampere count method is proven.

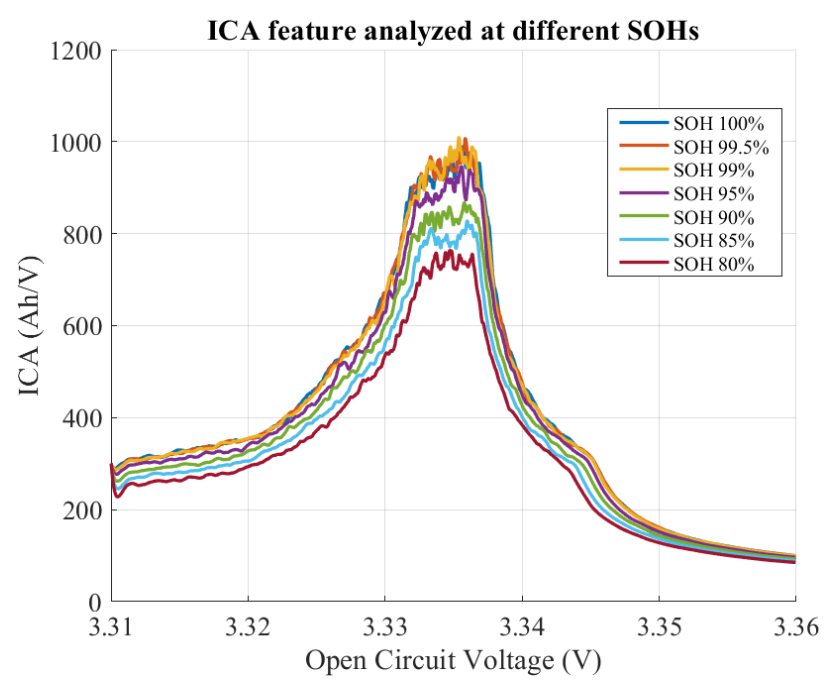

Fig. 9. Comparison fitered ICA features at different cell SOHs.

TABLE III

SOH ESTIMATION USING IMPROVED AMPERE-COUNT ALGORITHM: FOR EACH SOH DEFINED ARE PERFORMED 100 TESTS ON CELL THERMO-ELECTRIC MODEL WHICH SIMULATES REAL CALB CELL

\begin{tabular}{|l|l|}
\hline \multicolumn{1}{|c|}{ SOH original } & Capacity estimated Ah (SOH \%) \\
\hline $100 \%$ (Calibration test) & 20.1215 \\
$100 \%$ & $20.1224(100.0047 \pm 0.0363 \%)$ \\
$99.5 \%$ & $20.0404(99.5971 \pm 0.0369 \%)$ \\
$99 \%$ & $19.9319(99.0578 \pm 0.0428 \%)$ \\
$95 \%$ & $19.0583(94.7161 \pm 0.0383 \%)$ \\
$90 \%$ & $17.9575(89.2455 \pm 0.0352 \%)$ \\
$85 \%$ & $16.8606(83.7939 \pm 0.0319 \%)$ \\
$80 \%$ & $15.7574(78.3114 \pm 0.0283 \%)$ \\
\hline
\end{tabular}

\section{CONCLUSIONS}

An improved Ampere Count method, based on Incremental Capacity Analysis, for battery SOH estimation was carried out in this paper. $\mathrm{SOH}$ is defined only as ratio between actual capacity residual and capacity of a new cell (1). CALB (LiFePO4) cell is tested to verify algorithm's performance. Initially, electrical and thermal properties of lithium cell are described. Then, its same dynamics are simulated by cell thermo-electrical model implemented and realized on Siemens Amesim. Parameter of this model are sized to simulate not only same physical properties of real CALB cell, but to simulate the same degradation curve of capacity during its cycle life. After this, an improved Ampere-Count method was presented to estimate $\mathrm{SOH}$, based on Incremental Capacity Analysis on a particular feature of cell Ah-V profile. Feature analyzed is usually found in a SOC range (for CALB [66, 94] \%) which is often reachable along a common EV cycle. To enhance this method, a $2^{\text {nd }}$ order Butterworth filter was used. Algorithm for $\mathrm{SOH}$ estimation was implemented in Matlab. This algorithm was tested during simulations of complete recharge of cell thermo-electrical model on Amesim at different $\mathrm{SOH}$. Results, which are shown in Fig. III, prove 
that the aged cell shows a lower capacity accumulated than the new cell at the same voltage difference, confirming the aging phenomena caused by the electro-chemical behaviors of anode and electrolyte of the cell leading to a degradation process of active materials exchange between anodeelectrolyte interfaces. Moreover results in Table III verify the effectiveness of proposed method. This novel estimation $\mathrm{SOH}$ approach is innovative and it allows battery SOH online estimation and on board the vehicle. Indeed ISO and IEC battery capacity test standard requires to perform a complete recharge and discharge cycle for a correct rated capacity measurement. Using improved Ampere count method rated capacity should be simply estimated from so-called partial recharge made by the vehicle during the week. Moreover, with this new algorithm, it is possible to obtain an up-to-date estimation of $\mathrm{SOH}$ frequently, more times a week. Finally this method should be used during the entire cycle life of the battery. Indeed the $\mathrm{Ah}-\mathrm{V}$ curve feature highlighted in Fig. 1, in Fig. 6 and analyzed in this work is not sensitive respect to battery $\mathrm{SOH}$, so during entire its cycle life. However, improved Ampere-Count method elaborates an accurate $\mathrm{SOH}$ estimation if OCV and current values during phase recharge are accurately measured. In this work, accuracy voltage and current measurements are respectively supposed under $1 \%$ of cell nominal voltage and capacity. Moreover measurements are sampled in order of second. Possible future developments are certainly implementation of this algorithm in real industrial control systems for Lithium-Ion battery, as BMS, with appropriate current and voltage sensors, and finally the integration of this algorithm with other filter which computes battery parameters in different time scales (for example SOC, OCV, etc.).

\section{ACKNOWLEDGMENT}

The present work is using data coming from Gruppo Pretto company (Ponsacco, Italy); our special thanks to the leader of this company Alessandro Pretto for his support and collaboration.

\section{REFERENCES}

[1] ISO 12405-1, Electrically propelled road vehicles test specification for lithium-ion traction battery packs and systems Part 1: high-power applications, 2011.

[2] ISO 12405-2, Electrically propelled road vehicles test specification for lithium-ion traction battery packs and systems Part 2: high-energy applications 2012

[3] IEC 62660-1, "Secondary lithium-ion cells for the propulsion of electric road vehicles - Part 1: Performance testing", 2010.

[4] Elie Riviere, Pascal Venet, Ali Sari, Frederic Meniere, YannBultel, 'LiFePO4 Battery State of Health Online Estimation Using Electric Vehicle Embedded Incremental Capacity Analysis", 2015 IEEE Vehicle Power and Propulsion Conference (VPPC), 19-22 October 2015, Montreal, QC, Canada.

[5] Le D, Tang XD. , "Lithium-ion battery state of health estimation using ah- $v$ characterization", in: Annual conference of the prognostics and health management society; 2011. p. 367-73.

[6] P.Ruetschi, "Aging mechanisms and service life of lead-acid batteries", Journal of Power Sources Volume 127, Issues 1-2, 10 March 2004, Pages 33-44.

[7] R. Revel, J. Bernard, A. Delaille, P. Gyan, "Studies and modeling of the calendar aging of HEVs and EVs Li-ion cells: Simcal project", Batteries 2012.
[8] E. Prada, M. Petit, "Aging modeling for advanced Li-ion battery pack sizing and management for HEV/EV through LMS Amesim simulation platform", LMS Vehicle Conference France 2013.

[9] E. Locorotondo, L. Pugi, L. Berzi, M. Pierini, G. Lutzemberger, "Online identification of Thevenin equivalent circuit model parameters and estimation State Of Charge of Lithium-Ion batteries", in press.

[10] C. Zhang, K. Li, J. Deng and S. Song, "Improved Realtime State-ofCharge Estimation of LiFePO4 Battery Based on a Novel Thermoelectric Model" in IEEE Transactions on Industrial Electronics, vol. 64, no. 1, pp. 654-663, Jan. 2017.

[11] Lin X., Stefanopoulous A. G., Perez H. E., Siegel J.B., Li Y., Anderson R. D., "Quadruple adaptive observer of the core temperature in cylindrical Li-ion batteries and their health monitoring", in 2012 American Control Conference, ACC 2012 (pp. 578-583).

[12] S. Sepasi, R. Ghorbani and B. Y. Liaw, "SOC estimation for aged lithium-ion batteries using model adaptive extended Kalman filter", 2013 IEEE Transportation Electrification Conference and Expo (ITEC), Detroit, MI, 2013, pp. 1-6. 